\title{
Ultra-Wideband Geo-Regioning: A Novel Clustering and Localization Technique
}

\author{
Christoph Steiner, ${ }^{1}$ Frank Althaus, ${ }^{2}$ Florian Troesch, ${ }^{1}$ and Armin Wittneben ${ }^{1}$ \\ ${ }^{1}$ Communication Technology Laboratory, Department of Information Technology and Electrical Engineering, \\ ETH Zurich, $\mathrm{CH}-8092$ Zurich, Switzerland \\ ${ }^{2}$ International Electronics \& Engineering (IEE), S.A., L-5326 Contern, Luxembourg
}

Correspondence should be addressed to Christoph Steiner, steinech@nari.ee.ethz.ch

Received 1 March 2007; Revised 19 July 2007; Accepted 17 October 2007

Recommended by Sinan Gezici

\begin{abstract}
Ultra-wideband (UWB) technology enables a high temporal resolution of the propagation channel. Consequently, a channel impulse response between transmitter and receiver can be interpreted as signature for their relative positions. If the position of the receiver is known, the channel impulse response indicates the position of the transmitter and vice versa. This work introduces UWB geo-regioning as a clustering and localization method based on channel impulse response fingerprinting, develops a theoretical framework for performance analysis, and evaluates this approach by means of performance results based on measured channel impulse responses. Complexity issues are discussed and performance dependencies on signal-to-noise ratio, a priori knowledge, observation window, and system bandwidth are investigated.
\end{abstract}

Copyright (c) 2008 Christoph Steiner et al. This is an open access article distributed under the Creative Commons Attribution License, which permits unrestricted use, distribution, and reproduction in any medium, provided the original work is properly cited.

\section{INTRODUCTION}

Ultra-wideband (UWB) technology is characterized by relative bandwidths larger than $20 \%$ and absolute bandwidths of more than $500 \mathrm{MHz}$. These wide bandwidths improve the reliability of communications systems through frequency diversity and the accuracy of positioning techniques through the high temporal resolution of the propagation channel. The most promising UWB localization approaches exploiting the wide bandwidth are based on time of arrival estimation $[1,2]$, where the unknown position of a transmitter is calculated by trilateration using the estimated distances to, at least, three reference receivers with known positions. These methods work very accurately under line-ofsight (LOS) conditions. However, a general problem of localization and tracking systems using time of arrival estimates is the performance degradation under non-LOS conditions, since, the strongest and/or first arriving path may not correspond to the direct path $[3,4]$, yielding positively biased distance estimates.

A different localization paradigm is based on comparing a fingerprint or signature extracted from the received signal to entries in a database. A priori information is re- quired to generate this database. Possible types of fingerprint information are, for example, received signal strength (RSS), angular power profile, or power delay profile. Implemented indoor positioning systems based on RSS fingerprints and WLAN technology like RADAR [5] or EKAHAU achieve position estimation errors of less than $5 \mathrm{~m}$ for $75 \%$ of all classification cases using RSS measurements at three distributed receivers as a location fingerprint. The accuracy of such systems can be increased by adding more receivers, which in turn increases the complexity and the amount of data exchange. In contrast to this distributed approach, it is possible to increase the accuracy by using more signal parameters as fingerprint information. For example, Nerguizian et al. [6] use the mean excess delay, rms delay spread, maximum excess delay, total received power, number of multipath components, power of the first path, and the arrival time of the first path as fingerprint information. These parameters are extracted out of wideband channel measurements with $200 \mathrm{MHz}$ bandwidth. The fingerprints and the corresponding positions of the transmitter are used to train a neural network. The authors report position estimation errors of less than $2 \mathrm{~m}$ for $80 \%$ of the classification cases. 


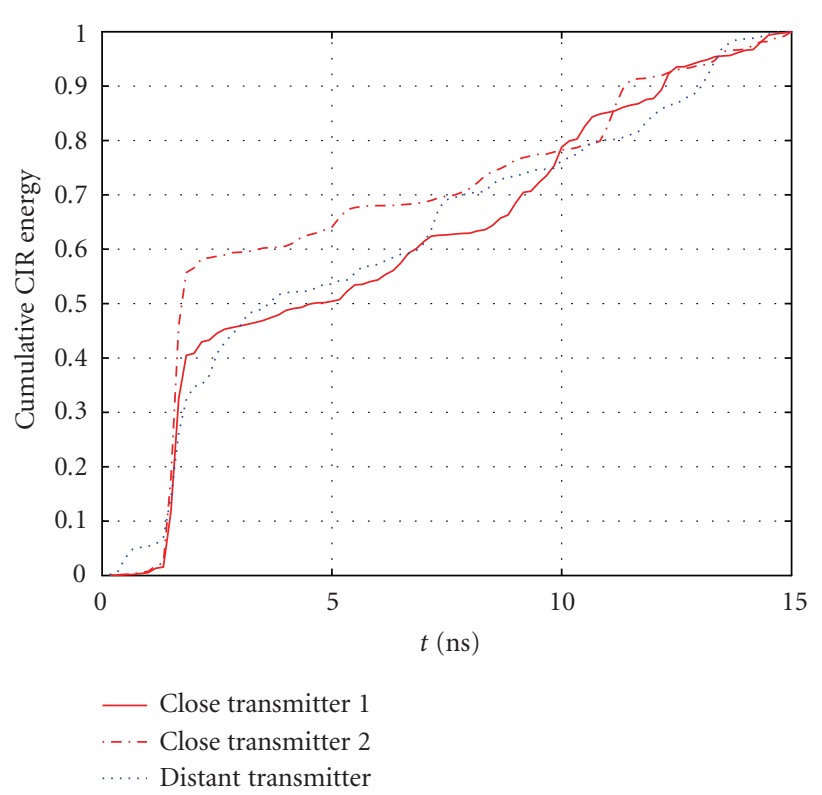

FIGURE 1: Normalized cumulative energy of CIRs from two close transmitters and one transmitter located far away from these two transmitters.

We show in this paper that communication systems with sufficiently high bandwidth can directly use the channel impulse response (CIR) as fingerprint information. We refer to this location fingerprinting method as UWB geo-regioning. Only one receiver is required and the whole computational complexity is shifted to this receiver such that the transmitters can be low-cost devices and need no additional signal processing or hardware. Moreover, no time synchronization between transmitter and receiver is necessary. Therefore, this is a very appealing technique for sensor networks, where the sensors are energy limited and the receiver (access point) is connected to a power supply allowing for more complexity. Energy-efficient coding techniques like distributed source coding or location aware routing protocols are enabled by UWB geo-regioning. An intelligent combination of geometric localization techniques and UWB geo-regioning can help to increase the accuracy and the robustness of positioning in harsh environments.

This paper aims to show the principle feasibility of UWB geo-regioning. A theoretical framework based on probability theory and statistic is established for algorithm design and to get insight into the problem structure. Furthermore, this framework enables the investigation of the impact of system parameters on the performance. In order to show the feasibility and evaluate the performance, the developed algorithms are applied to measured CIRs. Figure 1 depicts the normalized cumulative energy of three measured CIRs from two close transmitters and one transmitter far away from these two. This plot illustrates the difficulty of the problem, since no clear differences and similarities of the curves are noticeable.

This paper is organized as follows. Section 2 discusses the probabilistic modeling of CIRs originating from the same region. Section 3 introduces the theoretical frame- work based on maximum likelihood parameter estimation and maximum likelihood decision algorithms. In Section 4, analytic expressions for the probabilities of misclassification are derived, and insight into the problem structure is given. Section 5 describes the channel measurement campaign, whose results are later on used to evaluate UWB georegioning. Measurement postprocessing steps are described in Section 6. Section 7 gives a detailed performance evaluation. The paper is concluded with Section 8, where the contributions are summarized and an outlook into further research is given.

\section{Notation}

All vectors are column vectors, $I$ is the identity matrix, $(\cdot)^{T}$ denotes transposition, and $(\cdot)^{H}$ denotes complex conjugate transposition. The operator $\mathrm{E}(\cdot)$ denotes expectation, trace $(\cdot)$ is the sum of the diagonal elements of a matrix, $|\cdot|$ is the determinant, and eig $(\cdot)$ calculates the eigenvalues of a matrix. An estimate for parameter $\theta$ is denoted as $\hat{\theta}$. $\mathcal{C} \mathcal{N}(\cdot)$ denotes the multivariate proper complex Gaussian probability density function (PDF).

\section{STATISTICAL REGION MODELING}

Observations or measurements can be regarded as realizations of a random variable. This probabilistic concept is very useful in describing physical phenomenons, which govern the behavior of a system. Furthermore, it makes the system mathematically tractable.

For UWB geo-regioning, the wireless propagation channel is interpreted as linear time-invariant system, which is fully described by its impulse response. Moreover, regions are characterized by a probability model for the discrete time CIRs of transmitters located within this region to a fixed receiver. The selection of the probability model is based on channel-modeling literature and the constraint of mathematical tractability.

A common assumption on the small-scale fading behavior of channel taps is that they are complex Gaussian distributed. The justification of this assumption is given by the central limit theorem. Many reflected and scattered partial waves from different directions superimpose at the receive antenna and contribute to one channel tap with varying amplitude and phase. If the number of partial waves is large enough, the central limit theorem can be applied and the resulting distribution of the channel tap can be approximated as Gaussian [7]. However, as the tap duration becomes smaller (approaching UWB), less partial waves contribute to one channel tap. This fact questions the applicability of the central limit theorem. In literature, there exist various studies on the tap statistics for UWB channels. For the UWB channel tap amplitudes, the Nakagami [8], Lognormal [9], and Weibull [10] distributions are proposed. However, also Rayleigh and Rice amplitude distributions arising from the complex Gaussian channel tap distribution are supported by some channel measurement campaigns [11]. The phase distribution is commonly assumed uniform between $-\pi$ and $\pi$ and not further considered. 
The big advantage of the Gaussian assumption is the mathematical simplicity. For example, maximum likelihood estimators and deciders are easily derived if the Gaussian modeling is pursued. Therefore, and since also results in literature favor Gaussian channel taps, we stick to this assumption and model the channel taps with a complex Gaussian distribution.

\section{UWB GEO-REGIONING ALGORITHMS}

In this section, a theoretical framework is developed to prove the feasibility and evaluate the performance of UWB georegioning. The goal is to classify a transmitter with unknown position to its region based on its CIR. It is not at all clear whether this classification can be done based on the probabilistic modeling of CIRs described in Section 2. Therefore, we consider only two possible regions for the first analyis to get as much insight as possible. The presented algorithms can be easily extended to a general case of $M$ regions.

\subsection{Modeling assumptions and parameter estimation}

The discrete time samples of a CIR in complex baseband representation are modeled as proper complex Gaussian random vector with sample mean vector $\vec{\mu}$ and covariance matrix $\Sigma$. For a given region $A$, the mean and the covariance matrix are denoted as $\vec{\mu}_{A}$ and $\Sigma_{A}$, respectively. Thus the PDF of a CIR $\left(\vec{x}=[x[1], x[2], \ldots, x[K]]^{T}\right)$ with $K$ taps originating in region $A$ is given by $p(\vec{x} \mid A)=\mathcal{C} \mathcal{N}\left(\vec{\mu}_{A}, \Sigma_{A}\right)$. The model parameters $\vec{\mu}_{A}$ and $\Sigma_{A}$ are estimated from $N$, a priori known channel impulse responses from transmitters located within region $A$. The number of model parameters depends on $K$ and determines the amount of required a priori knowledge in order to get accurate parameter estimates. The maximum likelihood estimators using a set of $N>K$ independent CIR observations $\left\{\vec{x}_{A, 1}, \vec{x}_{A, 2}, \ldots, \vec{x}_{A, N}\right\}$ are given according to [12] by

$$
\begin{gathered}
\overrightarrow{\hat{\mu}}_{A}=\frac{1}{N} \sum_{i=1}^{N} \vec{x}_{A, i}, \\
\hat{\Sigma}_{A}=\frac{1}{N-1} \sum_{i=1}^{N}\left(\vec{x}_{A, i}-\overrightarrow{\hat{\mu}}_{A}\right)\left(\vec{x}_{A, i}-\overrightarrow{\hat{\mu}}_{A}\right)^{H} .
\end{gathered}
$$

The estimated mean $\overrightarrow{\hat{\mu}}_{A}$ is again complex Gaussian distributed, whereas the estimated covariance matrix $\hat{\Sigma}_{A}$ is distributed according to a Wishart distribution [12]. However, for the maximum likelihood decision algorithms in Section 3.2, these parameters are assumed to be deterministic, which is only true, if the sample size goes to infinity $(N \rightarrow \infty)$.

\section{Simplifying modeling assumptions}

(i) Zero mean assumption. The sample mean, averaged over many realizations of a CIR, where the transmitter, receiver, and environment are completely static, is just a noise-averaged version of the CIR itself. However, if the CIRs are averaged over different locations of the transmitter, the constructive and destructive interferences of the multipath reflections cancel themselves on average. If it is assumed that the sample mean vector is zero $\left(\vec{\mu}_{A}=\overrightarrow{0}\right)$, the maximum likelihood estimator for the covariance matrix changes to

$$
\widehat{\Sigma}_{0, A}=\frac{1}{N} \sum_{i=1}^{N}\left(\vec{x}_{A, i}\right)\left(\vec{x}_{A, i}\right)^{H} .
$$

(ii) Independent Tap Assumption. A significant reduction of model parameters is possible if statistically independent channel taps are assumed, which implies diagonal covariance matrices. This assumption follows from the widely used uncorrelated scattering assumption in channel modeling literature.

The complexity impacts of these simplifying assumptions on the maximum likelihood algorithms are discussed in Section 3.2, and the performance impacts are discussed in Section 7.

\subsection{Maximum likelihood algorithms}

This section derives the maximum likelihood decision algorithms for the binary hypotheses testing problem accounting for the different modeling assumptions. The maximum likelihood decision between hypotheses $A$ and $B$ with equal a priori probabilities is given by $p(\vec{x} \mid A) \underset{B}{\gtrless} p(\vec{x} \mid B)$, where $\vec{x}$ is a CIR of a transmitter with unknown region. For the general probability model, this decision rule reduces to

$$
\begin{aligned}
& \left(\vec{x}-\overrightarrow{\hat{\mu}}_{B}\right)^{H} \hat{\Sigma}_{B}^{-1}\left(\vec{x}-\overrightarrow{\hat{\mu}}_{B}\right)-\left(\vec{x}-\overrightarrow{\hat{\mu}}_{A}\right)^{H} \hat{\Sigma}_{A}^{-1}\left(\vec{x}-\overrightarrow{\hat{\mu}}_{A}\right) \\
& \underset{B}{A} \ln \left(\frac{\left|\hat{\Sigma}_{A}\right|}{\left|\hat{\Sigma}_{B}\right|}\right),
\end{aligned}
$$

where the decision threshold $\delta_{A B}$ is introduced as

$$
\delta_{A B}=\ln \left(\frac{\left|\hat{\Sigma}_{A}\right|}{\left|\hat{\Sigma}_{B}\right|}\right) .
$$

The complexity of this decision algorithm grows quadratic with the number of samples $\left(\mathcal{O}\left(K^{2}\right)\right)$. This algorithm is referred to as a covariance (COV) approach because correlations among channel taps are assumed and the full covariance matrix must be estimated.

\section{Simplifying modeling assumptions}

(i) Zero mean assumption. If it is assumed that the sample mean vector is zero $(\vec{\mu}=\overrightarrow{0})$, the maximum likelihood decision simplifies to

$$
\vec{x}^{H}\left(\hat{\Sigma}_{0, B}^{-1}-\hat{\Sigma}_{0, A}^{-1}\right) \vec{x} \underset{B}{\gtrless} \delta_{A B},
$$

where the matrix $\Delta_{A B}=\widehat{\Sigma}_{0, B}^{-1}-\widehat{\Sigma}_{0, A}^{-1}$ is Hermitian since $\widehat{\Sigma}_{0, A}$ and $\hat{\Sigma}_{0, B}$ are Hermitian and so are their inverses. The complexity is reduced slightly since no mean vector must be estimated. However, the complexity of the estimation and decision process is still $\mathcal{O}\left(K^{2}\right)$. 
(ii) Independent Tap Assumption. If independent channel taps and a zero mean vector are assumed, the maximum likelihood decision simplifies to

$$
\sum_{k=1}^{K}|x[k]|^{2}\left(\frac{1}{\hat{\Sigma}_{0, B}[k, k]}-\frac{1}{\hat{\Sigma}_{0, A}[k, k]}\right) \underset{B}{\gtrless} \sum_{k=1}^{K} \ln \left(\frac{\hat{\Sigma}_{0, A}[k, k]}{\hat{\Sigma}_{0, B}[k, k]}\right) .
$$

Here, the receiver must know only the main diagonals of the covariance matrices, which are also known as power delay profiles. This assumption reduces the complexity of estimation and decision significantly to $\mathcal{O}(K)$. This algorithm is referred to as the power delay profile (PDP) approach.

In general, there can exist $M>2$ possible regions. In this case, all $M((M-1) / 2)$ pairwise maximum likelihood metrics must be computed such that a transmitter can be classified to one region. Thus the complexity of the algorithm deciding between $M$ regions and using CIRs with $K$ taps grows according to $\mathcal{O}\left(K^{2} M^{2}\right)$. The performance is upper bounded in this case by the summation of all binary error probabilities as a direct consequence of the union bound.

\section{THEORETICAL PERFORMANCE ANALYSIS}

In order to analyze the performance, analytic expressions for the two probabilities of misclassification $P_{e \mid A}=P\left(\vec{x}^{H} \Delta_{A B} \vec{x} \leq\right.$ $\left.\delta_{A B} \mid A\right)$ and $P_{e \mid B}=1-P\left(\vec{x}^{H} \Delta_{A B} \vec{x} \leq \delta_{A B} \mid B\right)$ must be derived. Consequently, the PDF of the quadratic Hermitian form $z=\vec{x}^{H} \Delta_{A B} \vec{x}$ is searched. The zero mean assumption is used here since the derivation becomes mathematically intractable in case of nonzero and not equal mean vectors. Hypothesis $A$ is assumed, whereas the PDF given hypothesis $B$ can be calculated equivalently.

\subsection{Reduction to diagonal Hermitian form}

The following derivations can be found in more detail in [13, Appendix B]. The first step is to whiten the complex Gaussian random vector $\vec{x}$. This is done by eigenvalue decomposition of the covariance matrix $\widehat{\Sigma}_{0, A}$ according to $\widehat{\Sigma}_{0, A}=U_{A} \Lambda_{A} U_{A}^{H}$, where the real valued and nonnegative eigenvalues are stored in $\Lambda_{A}$, and the corresponding eigenvectors in the columns of the unitary matrix $U_{A}$. Thus writing $\vec{w}=\Lambda_{A}^{-0.5} U_{A}^{H} \vec{x}$ renders the random vector $\vec{w}$ with zero mean and identity covariance matrix shown by

$$
\begin{aligned}
\mathrm{E}\left(\vec{w} \vec{w}^{H}\right) & =\Lambda_{A}^{-0.5} U_{A}^{H} \mathrm{E}\left(\vec{x} \vec{x}^{H}\right) U_{A} \Lambda_{A}^{-0.5} \\
& =\Lambda_{A}^{-0.5} U_{A}^{H} U_{A} \Lambda_{A} U_{A}^{H} U_{A} \Lambda_{A}^{-0.5}=I .
\end{aligned}
$$

With this linear transformation, the general Hermitian form becomes $z=\vec{w}^{H} \Theta_{A} \vec{w}$, where the matrix $\Theta_{A}=$ $\Lambda_{A}^{0.5} U_{A}^{H} \Delta_{A B} U_{A} \Lambda_{A}^{0.5}$ is again Hermitian and can be diagonalized according to $\Theta_{A}=V_{A} \Phi_{A} V_{A}^{H}$. The eigenvalues of $\Theta_{A}$, collected in the diagonal matrix $\Phi_{A}$, are real but not necessarily positive. With one more unitary transformation $\vec{v}=$ $V_{A}^{H} \vec{w}$, a diagonal quadratic form, that is, a weighted sum of i.i.d. exponential random variables, is obtained;

$$
z=\vec{v}^{H} \Phi_{A} \vec{v}=\sum_{k=1}^{K} \phi_{A}[k]|v[k]|^{2}
$$

The weights $\vec{\phi}_{A}=\left[\phi_{A}[1], \phi_{A}[2], \ldots, \phi_{A}[K]\right]^{T}$ given hypothesis $A$ depend on $\Delta_{A B}$ and $\hat{\Sigma}_{0, A}$, and can be calculated by $\vec{\phi}_{A}=\operatorname{eig}\left(\Lambda_{A}^{0.5} U_{A}^{H} \Delta_{A B} U_{A} \Lambda_{A}^{0.5}\right)$, whereas the weights given hypothesis $B$ depend on $\Delta_{A B}$ and $\hat{\Sigma}_{0, B}$, and can be calculated by $\vec{\phi}_{B}=\operatorname{eig}\left(\Lambda_{B}^{0.5} U_{B}^{H} \Delta_{A B} U_{B} \Lambda_{B}^{0.5}\right)$.

\subsection{Analytic probabilities of misclassification}

For the derivation of the error probabilities, it is assumed that all weights in $\vec{\phi}_{A}$ are mutually distinct but can have different signs. Therefore, the sum in (8) is split into a part collecting all positive and a part collecting all negative weights according to

$$
\begin{aligned}
& z_{1}=\sum_{k_{1}=1}^{K_{1}}\left|\phi_{A}\left[k_{1}\right]\right|\left|v\left[k_{1}\right]\right|^{2} \text { for } \phi_{A}\left[k_{1}\right]>0, \\
& z_{2}=\sum_{k_{2}=K_{1}+1}^{K}\left|\phi_{A}\left[k_{2}\right]\right|\left|v\left[k_{2}\right]\right|^{2} \text { for } \phi_{A}\left[k_{2}\right] \leq 0,
\end{aligned}
$$

with $z=z_{1}-z_{2}$ and independent random variables $z_{1}$ and $z_{2}$. The probability density function of $z_{1}$ under hypothesis $A$ is given by (cf. [14])

$$
\begin{aligned}
f_{z_{1} \mid A}\left(z_{1}\right)= & \begin{cases}\sum_{k_{1}=1}^{K_{1}} \frac{C_{1 \mid A}\left[k_{1}\right]}{\phi_{A}\left[k_{1}\right]} \exp \left(-\frac{z_{1}}{\phi_{A}\left[k_{1}\right]}\right) & \text { for } z_{1} \geq 0, \\
0 & \text { for } z_{1}<0,\end{cases} \\
& \text { where } C_{1 \mid A}\left[k_{1}\right]=\prod_{i=1}^{K_{1}} \frac{\phi_{A}\left[k_{1}\right]}{\phi_{A}\left[k_{1}\right]-\phi_{A}[i]} .
\end{aligned}
$$

The PDF of $z_{2}$ is equivalent to (10) using the corresponding weights. Thus the probability of misclassification $P_{e \mid A}$ under hypothesis $A$ is given by

$$
\begin{aligned}
& \text { if } \delta_{A B}>0 \text {, } \\
& P_{e \mid A}=\int_{z_{2}=0}^{\infty} \int_{z_{1}=0}^{\delta_{A B}+z_{2}} f_{z_{1} \mid A}\left(z_{1}\right) f_{z_{2} \mid A}\left(z_{2}\right) \mathrm{d} z_{1} \mathrm{~d} z_{2} \\
& =\sum_{k_{1}=1}^{K_{1}} \sum_{k_{2}=K_{1}+1}^{K} C_{1 \mid A}\left[k_{1}\right] C_{2 \mid A}\left[k_{2}\right] \\
& \times\left(1-\exp \left(\frac{-\delta_{A B}}{\phi_{A}\left[k_{1}\right]}\right) \frac{\phi_{A}\left[k_{1}\right]}{\phi_{A}\left[k_{1}\right]+\phi_{A}\left[k_{2}\right]}\right), \\
& P_{e \mid A}=\int_{z_{2}=-\delta_{A B}+z_{1}}^{\infty} \int_{z_{1}=0}^{\infty} f_{z_{1} \mid A}\left(z_{1}\right) f_{z_{2} \mid A}\left(z_{2}\right) \mathrm{d} z_{1} \mathrm{~d} z_{2} \\
& =\sum_{k_{1}=1}^{K_{1}} \sum_{k_{2}=K_{1}+1}^{K} C_{1 \mid A}\left[k_{1}\right] C_{2 \mid A}\left[k_{2}\right] \\
& \times\left(\exp \left(\frac{\delta_{A B}}{\phi_{A}\left[k_{2}\right]}\right) \frac{\phi_{A}\left[k_{2}\right]}{\phi_{A}\left[k_{1}\right]+\phi_{A}\left[k_{2}\right]}\right) .
\end{aligned}
$$

The decision threshold $\delta_{A B}$ can be expressed in terms of the weights $\vec{\phi}_{A}$ and $\vec{\phi}_{B}$ according to

$$
\delta_{A B}=\ln \left(\frac{\left|\hat{\Sigma}_{0, A}\right|}{\left|\hat{\Sigma}_{0, B}\right|}\right)=\ln \left(\frac{\left|\Lambda_{A}\right|}{\left|\Lambda_{B}\right|}\right)=\ln \left(\frac{\left|\Phi_{A}\right|}{\left|\Phi_{B}\right|}\right),
$$


where the first equality is obvious due to eigenvalue decomposition and the last equality is proven by

$$
\begin{aligned}
& \left|\Phi_{A}\right|=\left|\Lambda_{A}^{0.5} U_{A}^{H} \Delta_{A B} U_{A} \Lambda_{A}^{0.5}\right|=\left|\Lambda_{A}\right|\left|\Delta_{A B}\right|, \\
& \left|\Phi_{B}\right|=\left|\Lambda_{B}^{0.5} U_{B}^{H} \Delta_{A B} U_{B} \Lambda_{B}^{0.5}\right|=\left|\Lambda_{B}\right|\left|\Delta_{A B}\right|,
\end{aligned}
$$

and taking the fraction of $\left|\Phi_{A}\right|$ and $\left|\Phi_{B}\right|$.

\subsection{Asymmetric decision problem}

The equations for the probabilities of misclassification $P_{e \mid A}$ in (11) and $P_{e \mid B}$ have, in general, different parameters under each hypothesis determined by the corresponding weight vectors $\vec{\phi}_{A}$ and $\vec{\phi}_{B}$. Therefore, the considered decision problem is asymmetric, meaning that, in general, $P_{e \mid A} \neq P_{e \mid B}$. This can be visualized if the dimensionality of the CIR is reduced to one sample, that is, $K=1$. If $\phi_{A}>0$ and $\phi_{B}>0$, the PDFs reduce to

$$
\begin{aligned}
& f_{z \mid A}(z)= \begin{cases}\frac{1}{\phi_{A}} \exp \left(-\frac{z}{\phi_{A}}\right) & \text { for } z \geq 0, \\
0 & \text { for } z<0,\end{cases} \\
& f_{z \mid B}(z)= \begin{cases}\frac{1}{\phi_{B}} \exp \left(-\frac{z}{\phi_{B}}\right) & \text { for } z \geq 0, \\
0 & \text { for } z>0,\end{cases}
\end{aligned}
$$

where $\phi_{A}=\hat{\Sigma}_{0, A} / \widehat{\Sigma}_{0, B}-1, \phi_{B}=1-\hat{\Sigma}_{0, B} / \widehat{\Sigma}_{0, A}$, and $\delta_{A B}=$ $\ln \left(\widehat{\Sigma}_{0, A} / \widehat{\Sigma}_{0, B}\right)$. Thus $P_{e \mid A}=1-\exp \left(-\delta_{A B} / \phi_{A}\right)$ and $P_{e \mid B}=$ $\exp \left(-\delta_{A B} / \phi_{B}\right)$. Setting $\hat{\Sigma}_{0, A}=3$ and $\hat{\Sigma}_{0, B}=1$ gives a decision threshold at $\delta_{A B}=\ln (3)$ and error probabilities of $P_{e \mid A} \approx 0.42$ and $P_{e \mid B} \approx 0.19$.

\section{UWB CHANNEL MEASUREMENT CAMPAIGN}

A CIR measurement campaign tailored to the verification and performance analysis of UWB geo-regioning has been performed and is summarized in the following. A thorough description can be found in [15].

\subsection{Measurement setup}

The measurements have been performed at ETH Zurich in a big cellar room (cf. Figure 2) with a size of about $7.4 \mathrm{~m} \times$ $15 \mathrm{~m}$ and a height of $6 \mathrm{~m}$. There are many metallic objects in the room as, for example, metallic shelves, heating pipes, cabinets, and metal cores, implying a rich multipath environment.

A time-domain correlation method is used to measure the CIRs. The principle is to perform a cross correlation between the received signal and the transmit signal known at the receiver. In practice, the transmit signal is often generated using pseudorandom bit sequences or $\mathrm{m}$-sequences. The transmit signal is fed to a power amplifier and finally to the transmit antenna. The signal propagates through the channel, is received by the receive antenna, and is sampled by a real-time sampling scope with a sampling frequency of $20 \mathrm{GHz}$. The measurement frequency range is roughly limited from $3 \mathrm{GHz}$ to $6 \mathrm{GHz}$ by the transfer function of the

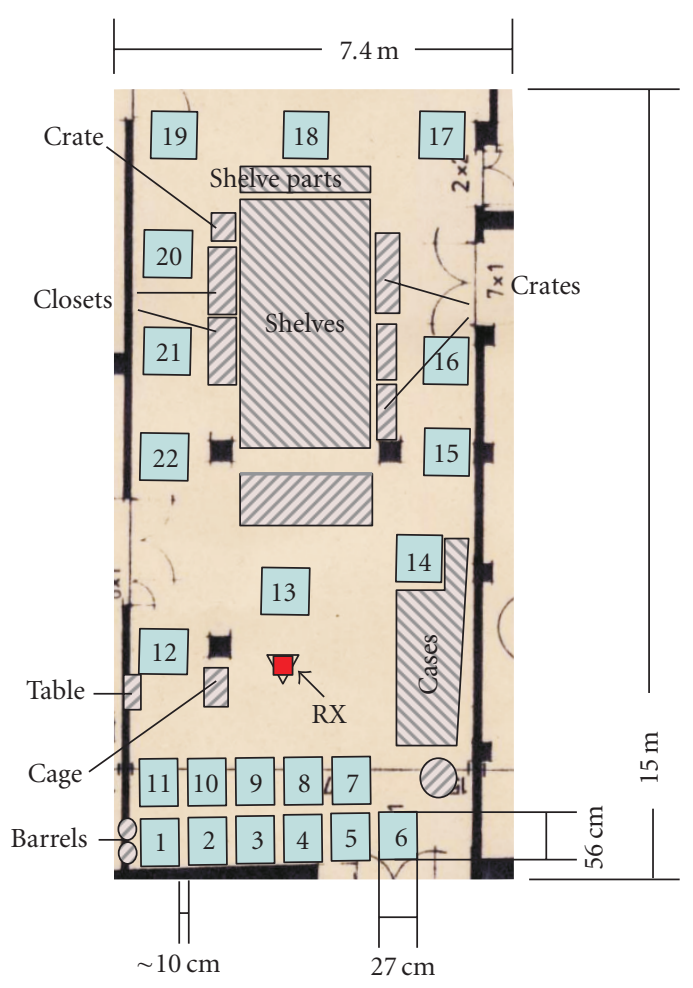

FIgURE 2: Marked regions in the cellar room.

UWB antenna and the cut-off frequency of the amplifier. The reference signal for the cross correlation is stored in the scope such that no wired connection between the transmitter and the receiver is required. This means that the absolute temporal delays of the CIRs are unknown. Furthermore, the impulse responses of the transmit and receive antennae are comprised in the measured CIRs.

\subsection{Measurement scenario}

The goal of this campaign has been to collect a sufficient number of CIR measurements for one static receiver and a moving transmitter located in 22 predefined regions with a size of $27 \mathrm{~cm} \times 56 \mathrm{~cm}$ (cf. Figure 2). The maximum distance between two regions is approximately $16 \mathrm{~m}$, whereas the minimum separation of two transmitter positions in two different regions is approximately $10 \mathrm{~cm}$.

The transmitter is moved with an almost constant speed of $1 \mathrm{~cm} / \mathrm{s}$ within each region. The trigger at the scope is not synchronized with the movement of the transmitter, which means that the exact positions of the transmitter within each region are unknown. However, since triggering is done periodically every 1.7 seconds due to hardware limitations, the spacing of subsequent measured CIRs is approximately $1.7 \mathrm{~cm}$. In total, $600 \mathrm{CIRs}$ per region have been measured.

\section{MEASUREMENT DATA POSTPROCESSING}

Before the measurements can be used for the evaluation of UWB geo-regioning, there are several postprocessing steps 


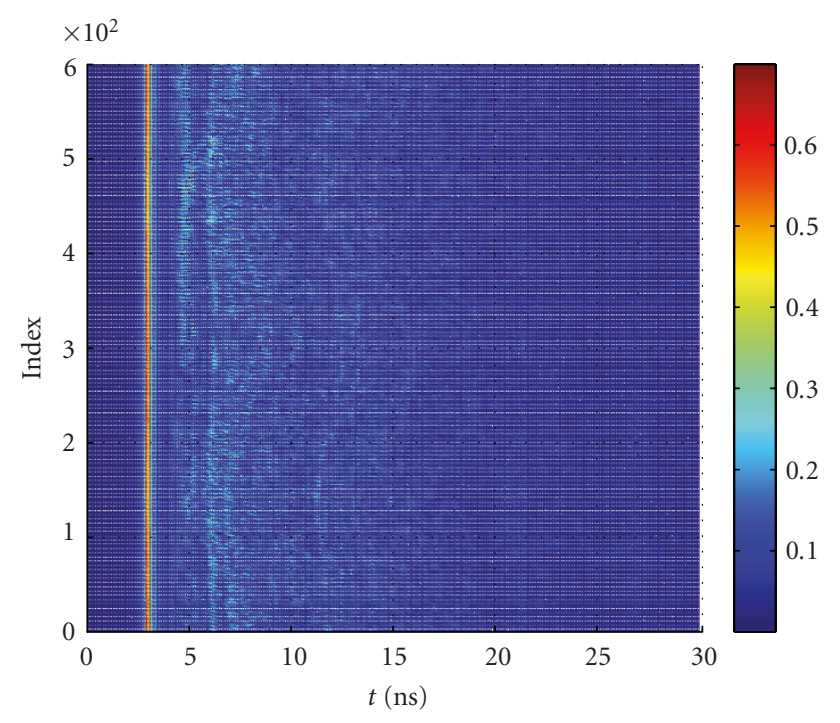

(a)

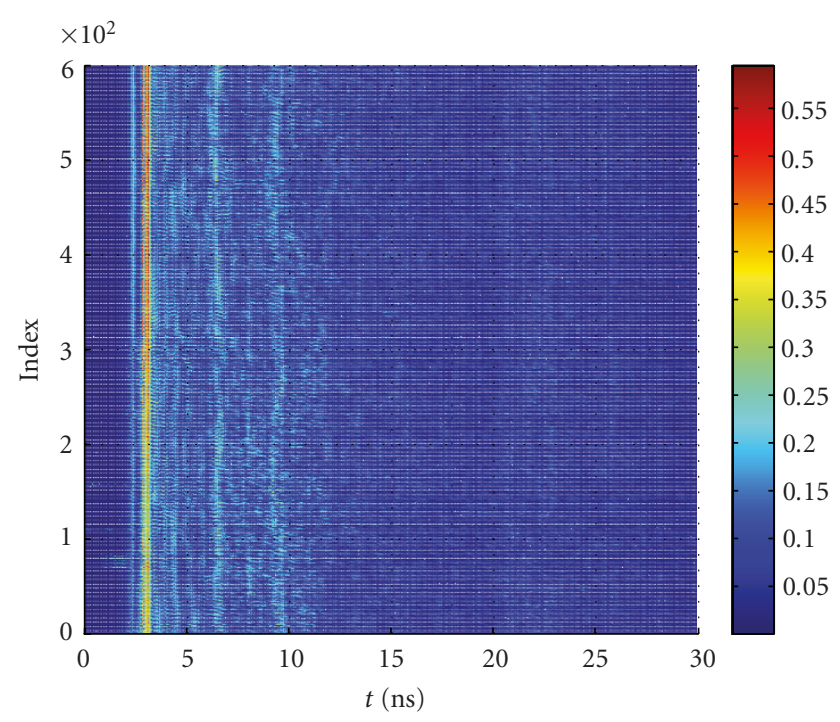

(b)

FIGURE 3: 600 measured and postprocessed CIRs (absolute values) for LOS region 4 (left) and non-LOS region 17 (right) in equivalent baseband representation and sampled at Nyquist rate.

necessary, which are explained as follows. The first step is to represent the measured passband data in equivalent baseband, implying complex channel taps. This can be done since the measurement frequency range is limited from 3 to $6 \mathrm{GHz}$ by hardware constraints. Figure 3 depicts absolute values of 600 postprocessed CIRs within regions 4 and 17.

\subsection{Alignment of the CIRs}

As stated, no absolute timing information is provided by the measurement procedure. Nevertheless, the measured CIRs must be aligned in time such that a meaningful statistical description can be extracted.

The strategy is to align the CIRs to a reference sample specified as their sample with the maximum absolute value. In order to achieve a higher resolution for the alignment, the CIRs are interpolated, and afterwards, the reference sample (maximum absolute value) is searched. The phase information is neglected for this alignment procedure. The aligned CIRs are down sampled such that tap correlations due to over sampling are removed.

In LOS situations, the CIRs are aligned to the direct path, implying that samples, before the reference sample, are just negligible noise samples. In case of a non-LOS situation, the CIRs are aligned to the strongest path, which is not necessarily the direct path. This means that also the samples before the reference sample can carry significant CIR energy and valuable information for UWB geo-regioning. There is a clear tradeoff between accounting for possible non-LOS situations and wasting samples. A heuristic approach used in the following cuts the section of a CIR to $\lfloor K / 10\rfloor$ taps before the reference sample and $K-\lceil K / 10\rceil$ samples after the reference sample, where $K$ is the total number of channel taps within the observation window.

\subsection{Measurement signal-to-noise ratio}

The measurement noise due to the electronics of the scope is assumed as an additive zero mean white Gaussian noise process. The noise samples are therefore Gaussian distributed with zero mean and variance $\sigma_{\text {mea }}^{2}$, which is given by the room temperature, the noise figure of the scope, and the measurement bandwidth. The measurement signal-to-noise ratio (SNR) is defined as the energy of the CIR over $\sigma_{\text {mea }}^{2}$ and ranges from 45 to $55 \mathrm{~dB}$ depending on the transmitter position. Parameter estimation in Section 3 and alignment are done at $\mathrm{SNR}_{\text {mea }}$.

\subsection{Energy normalization}

In general, it cannot be guaranteed that UWB transmitters use constantly the same transmit power. This happens, for example, when the data rate is adapted to the current work load or channel conditions. Moreover, the path-loss-modelbased distance estimates are rather unreliable in indoor and multipath environments. Therefore, the path loss information is neglected in the following investigations and the CIRs are normalized to energy one. However, a performance improvement can be expected, if the RSS information is used in addition.

\section{PERFORMANCE EVALUATION}

This section presents performance results in terms of $P_{e \mid A}$ and $P_{e \mid B}$ for UWB geo-regioning based on the algorithms derived in Section 3 and the measurements described in Section 5. Additionally, theoretical error probabilities are computed for CIRs, which are realizations of the modeling PDF. This means that the theoretical CIRs originating 


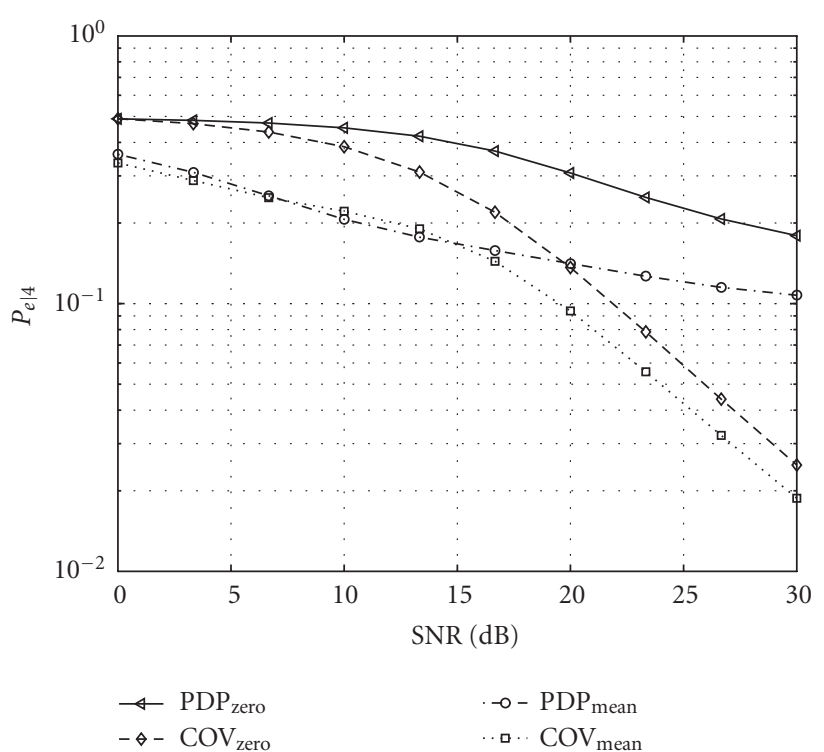

(a)

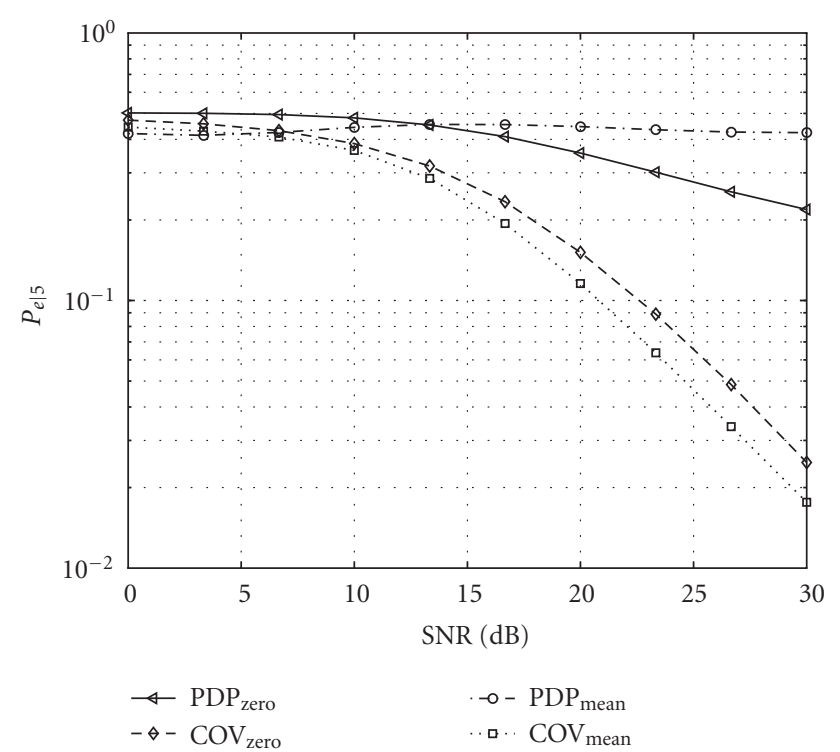

(b)

FIGURE 4: Region pair $(4,5)$ with measured CIRs.

from region $A$ are realizations of $\mathcal{C} \mathcal{N}\left(\overrightarrow{\hat{\mu}}_{A}, \hat{\Sigma}_{A}\right)$ in the general case and of $\mathcal{C} \mathcal{N}\left(\overrightarrow{0}, \hat{\Sigma}_{0, A}\right)$ in the zero mean case. If independent channel taps are assumed, the corresponding covariance matrices are diagonal. The theoretic results show fundamental performance limits and which modeling assumption matches the measured CIRs best.

Two representative region pairs are chosen out of the 22 measured regions. Regions 4 and 5 are two neighboring LOS regions and represent a worst-case scenario. It is expected that CIRs originating in these regions are quite similar and cannot be distinguished very well. On the other hand, regions 4 and 17 are about $16 \mathrm{~m}$ apart, and furthermore, region 17 is non-LOS representing a best-case scenario. Performance results in between these two extreme cases are achieved for the remaining region pairs. Additional performance results for algorithms based on the zero mean assumption are available in [16].

The following sections provide performance results for different design parameters. The outcomes show fundamental dependencies on the SNR, the number of a priori CIRs for parameter estimation, observation window, and system bandwidth.

\subsection{Signal-to-noise ratio}

In order to emulate different SNR operating points, the CIR under test $\vec{x}_{n}=\vec{x}+\vec{n}$ is corrupted by additive, proper, complex Gaussian, and i.i.d. noise samples $(\vec{n})$ with variance $\sigma^{2}$. The model for the PDF of the noisy CIR vector $\vec{x}_{n}$ originating from region $A$ is adapted to

$$
p\left(\vec{x}_{n} \mid A\right)=\mathcal{C N}\left(\overrightarrow{\hat{\mu}}_{A}, \hat{\Sigma}_{A}+\sigma^{2} I\right) .
$$

Accordingly, the SNR in $\mathrm{dB}$ is defined as

$$
\begin{aligned}
\mathrm{SNR} & =10 \log _{10}\left(\frac{\mathrm{E}\left(\sum_{i=1}^{K}|x[i]|^{2}\right)}{\sigma^{2}}\right) \\
& =10 \log _{10}\left(\frac{\sum_{i=1}^{K} \lambda[i]}{\sigma^{2}}\right) .
\end{aligned}
$$

The random variables $|x[i]|^{2}$ are noncentral chi-square distributed with two degrees of freedom and means $\lambda[i]=$ $\hat{\Sigma}[i, i]+|\hat{\mu}[i]|^{2}$ in general, or $\lambda_{0}[i]=\widehat{\Sigma}_{0}[i, i]$ in the zero mean case.

The 600 measured CIRs are partitioned into a set of size $N=400$ used for parameter estimation and the rest for error probability calculation. In order to calculate the expected probabilities of misclassification, a cross validation method [17] is used, where the error probabilities are averaged over 50 random partitions of estimation and test data. This method calculates values for $P_{e \mid A}$ and $P_{e \mid B}$ that can be expected if $400 \mathrm{CIRs}$ are used for parameter estimation. Since the estimated parameters are different for each random partition, also the theoretical results are averaged over the same random partitions in order to present a fair comparison.

The system bandwidth is $3 \mathrm{GHz}$, the observation window is $15 \mathrm{~ns}$, and the sampling frequency is $6 \mathrm{GHz}$. This means that a CIR vector consists of $K=90$ samples. For each CIR under test $10^{3}$, realizations of the noise vector $\vec{n}$ are generated and the error probabilities are averaged over the noise realizations. Figures 4 and 5 show probabilities of misclassification depending on the SNR for all presented algorithms in Section 3. The abbreviation COV indicates the covariance approach (Algorithm (3)) with nonzero mean (subscript mean) or algorithm (5) assuming zero mean (subscript zero). The abbreviation PDP indicates the power delay 


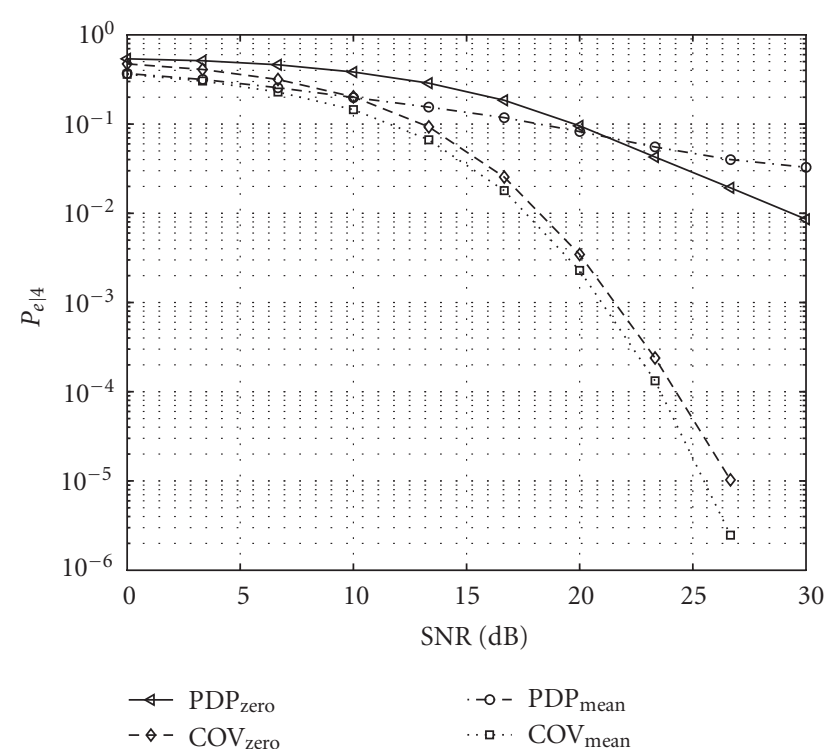

(a)

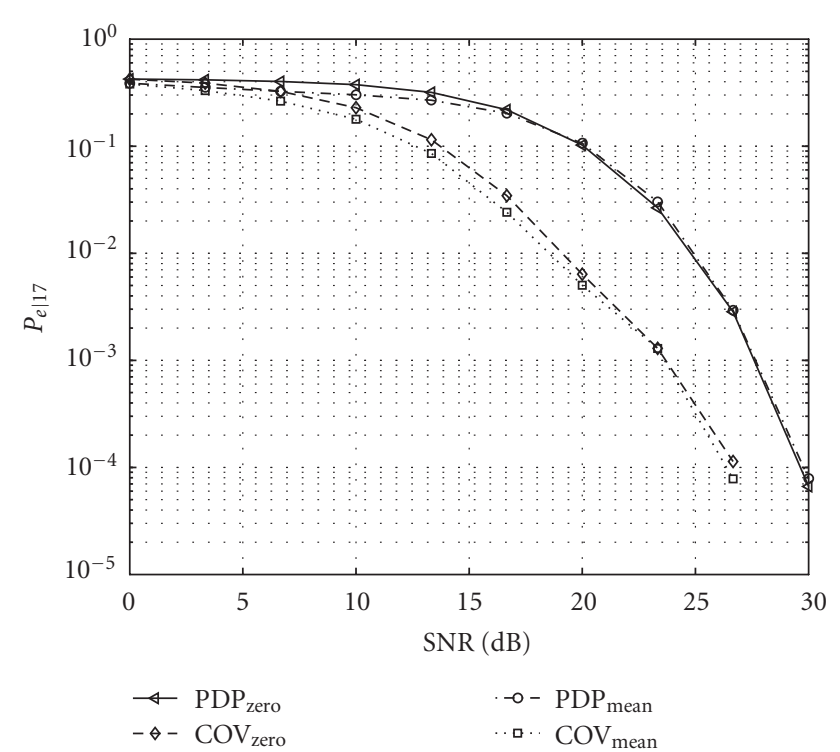

(b)

FIgURE 5: Region pair $(4,17)$ with measured CIRs.

profile approach (Algorithm (6)) with the same meaning of the subscripts.

As expected, it can be observed that CIRs from regions 4 and 5 are not as good distinguishable as CIRs from regions 4 and 17. Surprisingly, it is possible to achieve probabilities of misclassification of almost $10^{-2}$ even for regions 4 and 5 if the channel tap correlations are exploited (COV) and the SNR is larger than $30 \mathrm{~dB}$. In case of the independent taps assumption (PDP), the classification algorithms fail to achieve probabilities of misclassification of less than $10^{-1}$.

The classification algorithm assuming nonzero means and independent taps ( $\mathrm{PDP}_{\text {mean }}$ ) shows high-error probabilities and a very asymmetric behavior, especially in Figure 4, where two LOS regions are considered. The reason for this is a probability model mismatch for the taps around the reference sample, since their empirical tap distribution cannot be modeled by a complex Gaussian distribution. The $\mathrm{COV}_{\text {mean }}$ algorithm can compensate for this modeling mismatch by accounting also for the phases of these taps. It can be seen that the curves $P_{e \mid 5}$ in Figure 4 and $P_{e \mid 4}$ in Figure 5 for $\mathrm{PDP}_{\text {mean }}$ show better performance for low SNR and worse for high SNR than PDP zero . This happens because the CIRs, which have phases similar to the estimated mean, boost the performance in the low SNR regime. However, in the high SNR regime, the CIRs with phases different from the estimated mean dominate and limit the performance. In order to exploit the mean component in LOS situations, it would be necessary to modify the probability model for the taps around the reference sample.

Figures 6 and 7 depict performance results if theoretic CIRs are tested. By increasing the modeling complexity, SNR gains can be obtained. As expected, the COV algorithms outperform the PDP algorithms, but it is noticeable that the differences are much smaller compared to the performance results achieved with measured CIRs. It is also visible that the knowledge of the mean promises very good performance for the PDP algorithms. However, due to the mentioned modeling mismatch this cannot be exploited.

When comparing Figure 4 with Figure 6 and Figure 5 with Figure 7, it can be seen that accounting for correlated channel taps matches the expected theoretical results better. Additionally, the COV algorithms show, besides error floor reduction, also significant SNR gains in Figures 4 and 5. These gains are vital for UWB communication systems, which work generally in the low SNR regime due to transmit power restrictions. In order to achieve error probabilities of less than $10^{-2}$, an SNR in the range of $25 \mathrm{~dB}$ is required, which is rather high for UWB communications. However, a higher SNR can be expected for channel estimation compared to data detection because training sequences are used to average out the noise influence.

\subsection{A priori knowledge}

The number of a priori known CIRs per region $(N)$ plays an important role for UWB geo-regioning. Therefore, its impact on the performance is investigated here. For all subsequent results, PDP and COV algorithms assuming zeromean channel taps are used. Figures 8 and 9 depict averaged error probabilities over 50 cross validation iterations for increasing $N, 3 \mathrm{GHz}$ bandwidth, $K=90$ channel taps, and a 15 ns observation window. In the legend, Q90 denotes the $90 \%$ quantile, that is, 45 of the 50 random partitions $(90 \%)$ show smaller error probabilities than the Q90 curves.

It can be seen that the error probabilities decrease for increasing a priori knowledge due to more accurate parameter estimates. Moreover, the impact of increasing $N$ on the PDP algorithm is less significant because the number of parameters to estimate is here significantly smaller than that for the COV algorithm. The curve for $P_{e \mid 17}$ in Figure 9 shows 


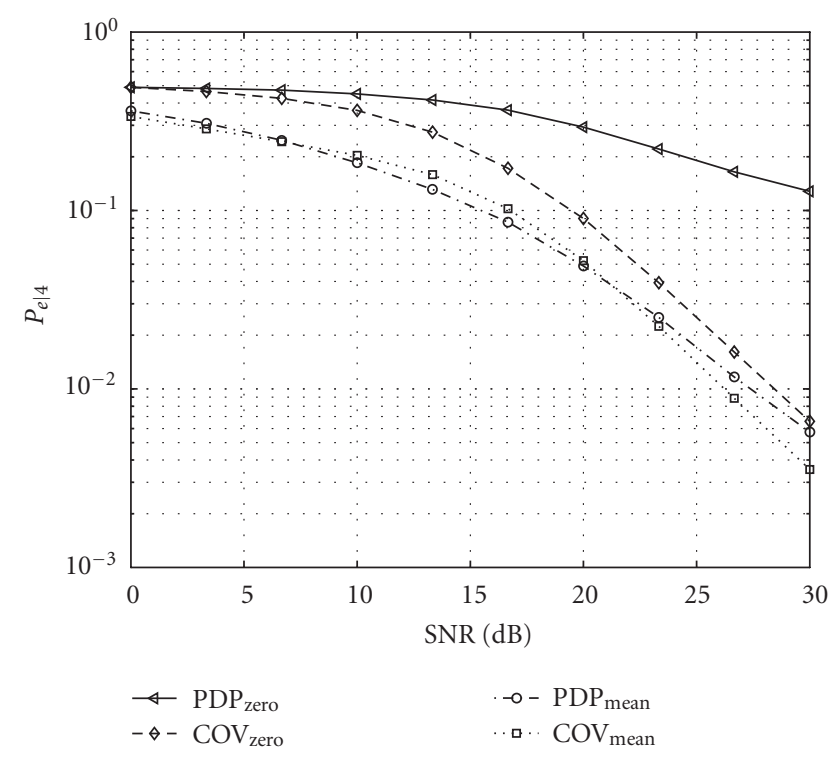

(a)

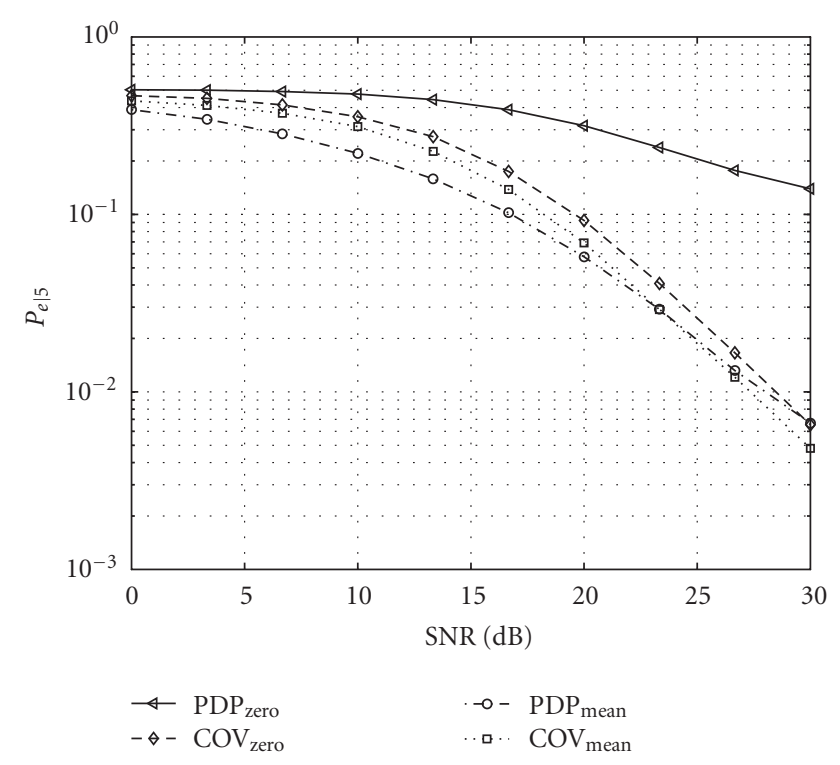

(b)

FIgURE 6: Region pair $(4,5)$ with theoretic CIRs.

a higher variation of the error probabilities and a reduced performance improvement for increasing $N$ compared to the curves for $P_{e \mid 4}$ in Figure 9. This is explained by the nature of region 17, which is non-LOS and not as coherent as region 4. This means that the temporal multipath patterns of CIRs within region 17 vary more when the position of the transmitter is changed.

Concluding, it is possible to achieve reasonable low-error probabilities for CIRs with $K=90$ channel taps, a randomly selected subset of $N \approx 200$ a priori CIRs per region, and exploiting the channel tap correlations. These results are very promising for bootstrap methods, which classify CIRs in a normal operating mode and use them after classification for parameter estimation to increase estimation accuracy. Whether the classified CIR is used for parameter estimation depends on a soft indicator like the likelihood value.

\subsection{Observation window}

In this section, the impact of the observation window on the performance is investigated. The observation window in the previous sections was set to $15 \mathrm{~ns}$, which is rather small for an indoor or industry scenario, where rms delay spreads of up to $50 \mathrm{~ns}$ are reported in literature $[8,18,19]$. Not all multipath components are captured within $15 \mathrm{~ns}$, suggesting that there is room for performance improvement by enlarging the observation window. However, the received energy of the multipath components falls below the noise floor after a time much shorter than $50 \mathrm{~ns}$ for practical UWB communication. Therefore, it is expected that the performance gain obtained by enlarging the observation window is limited by the SNR.

In Figure 10, the observation window is varied from 1 to $50 \mathrm{~ns}$, the bandwidth is $3 \mathrm{GHz}, N=400$ a priori CIRs are used, region pair 4 and 5 is considered, and the SNR is set to $25 \mathrm{~dB}$.
It is important to notice that the error probabilities depending on the observation window are region specific, in the sense that they depend on the temporal delay of significant multipath components. Nevertheless, it can be concluded that the PDP algorithm is almost insensitive to the increase of the observation window because the SNR per tap decreases as the number of taps increases. This means that the channel taps are governed by the additive noise samples. On the contrary, the COV algorithm shows performance improvements since the correlation between the taps is not affected by adding independent noise samples. However, the increase of the observation window implies a performance degradation due to the increased number of model parameters.

\subsection{System bandwidth}

The bandwidth determines the temporal resolution of multipath reflections with different propagation delays. By decreasing the bandwidth, the time-domain signal broadens and more and more reflections overlap in time. This means that signal contributions from different reflectors and scatterers cannot be distinguished from each other anymore. Since UWB geo-regioning relies on the ability to resolve the multipath components, it can be expected that the performance drops by decreasing the system bandwidth. However, a larger observation window can be covered with the same number of samples since Nyquist rate drops for a smaller bandwidth. Equivalently, a constant observation window can be covered with less CIR samples. In the following simulations, the observation window is set to $20 \mathrm{~ns}$ and the bandwidth is varied from $300 \mathrm{MHz}$ up to the full $3 \mathrm{GHz}$ measurement bandwidth, implying CIR lengths from $K=12$ up to $K=120$ samples. 


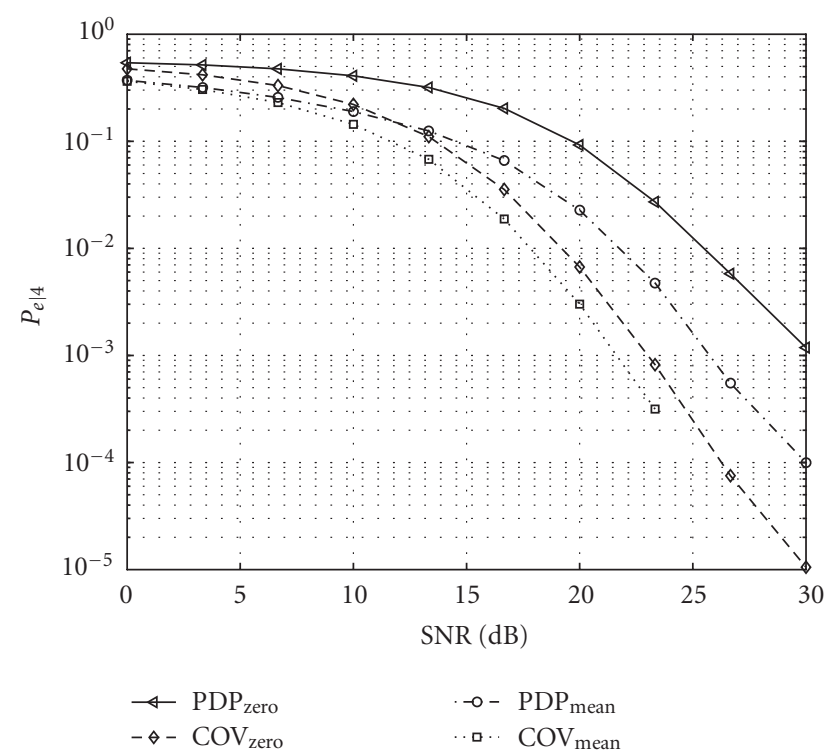

(a)

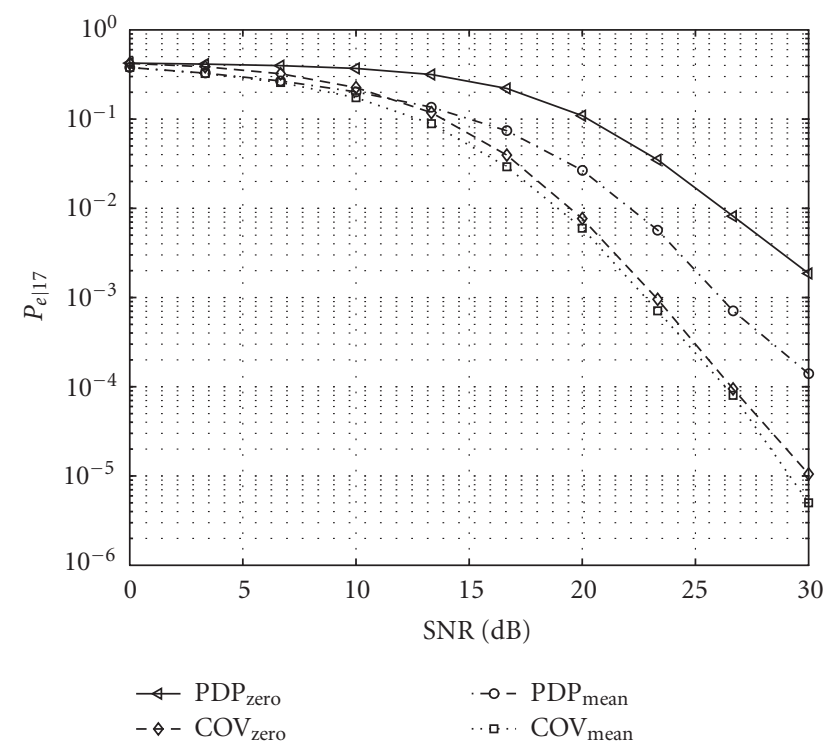

(b)

Figure 7: Region pair $(4,17)$ with theoretic CIRs.

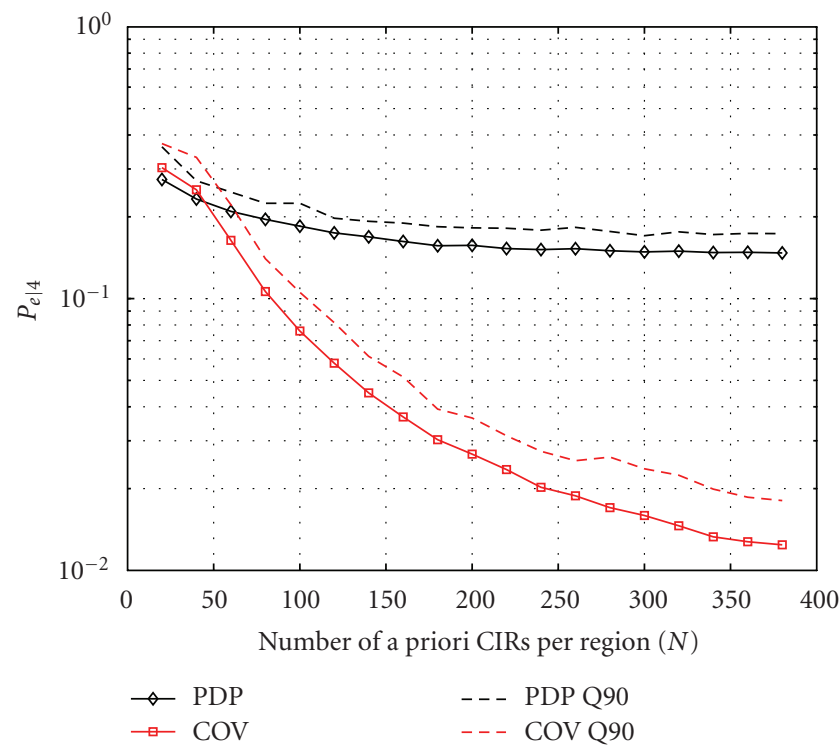

(a)

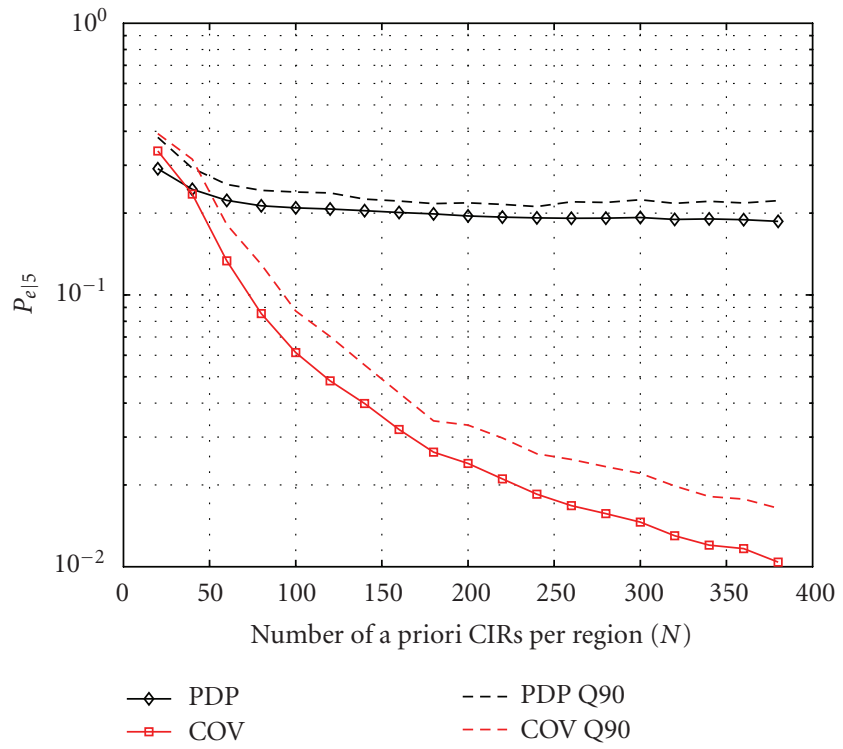

(b)

FIgURE 8: Region pair $(4,5)$ with measured CIRs at SNR $=35 \mathrm{~dB}$ for increasing $N$.

Figure 11 shows the error probabilities for region pair 4 and 5 at an SNR of $25 \mathrm{~dB}$ for measured (subscript mea) and theoretical (subscript theo) CIRs. There is a slight performance gain for the PDP algorithm for increasing bandwidths until $1.5 \mathrm{GHz}$. Beyond that, the higher temporal resolution does not help to distinguish CIRs from regions 4 and 5, if independent taps are assumed. In contrast, the COV algorithm shows a continuous improvement. The increasing gap between the results for theoretic and measured CIRs for COV is explained by the increasing parameter estimation error due to the increased number of channel taps.
From these results, it can be concluded that the expected performance dependency of UWB geo-regioning on the used bandwidth is present. Furthermore, it is evident that the amount of required a priori knowledge can be reduced by decreasing the bandwidth and consequently the number of model parameters.

\section{CONCLUSIONS AND OUTLOOK}

A novel clustering and localization technique based on CIR fingerprinting, named UWB geo-regioning, has been 


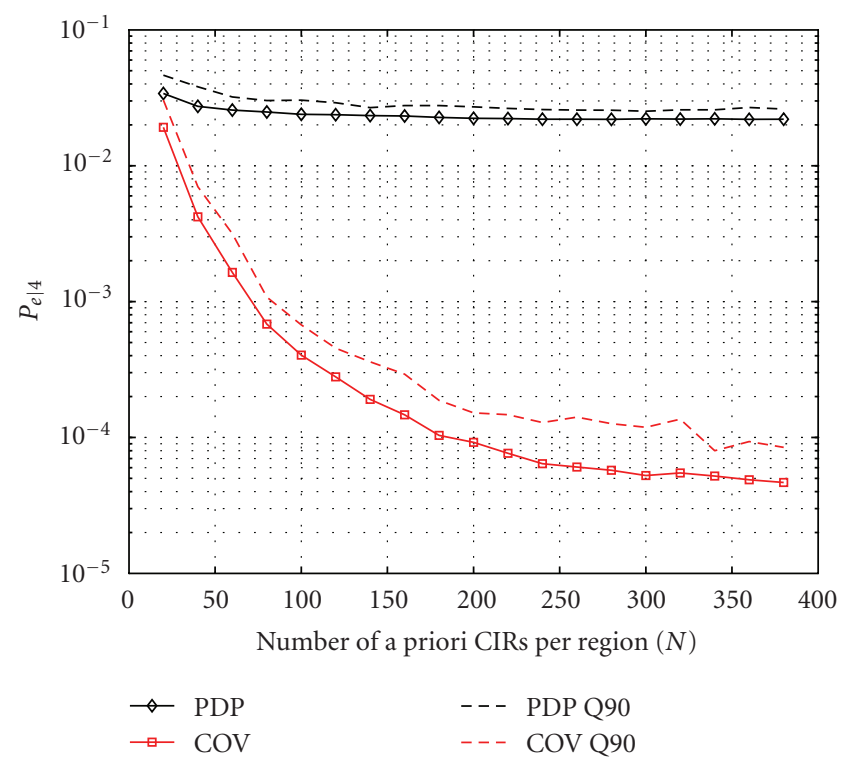

(a)

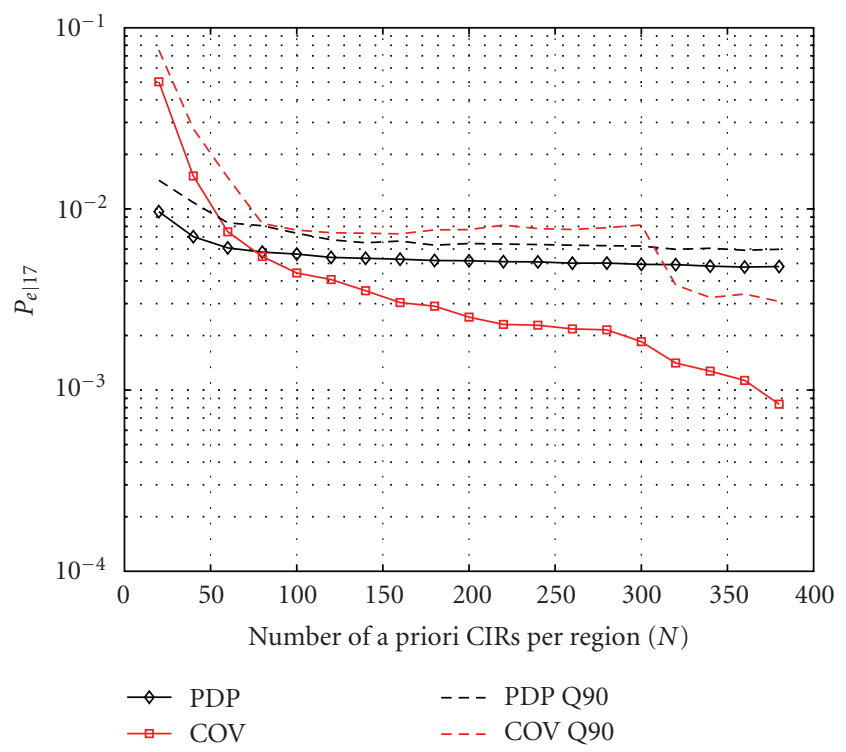

(b)

FIgURE 9: Region pair $(4,17)$ with measured CIRs at $\mathrm{SNR}=25 \mathrm{~dB}$ for increasing $N$.

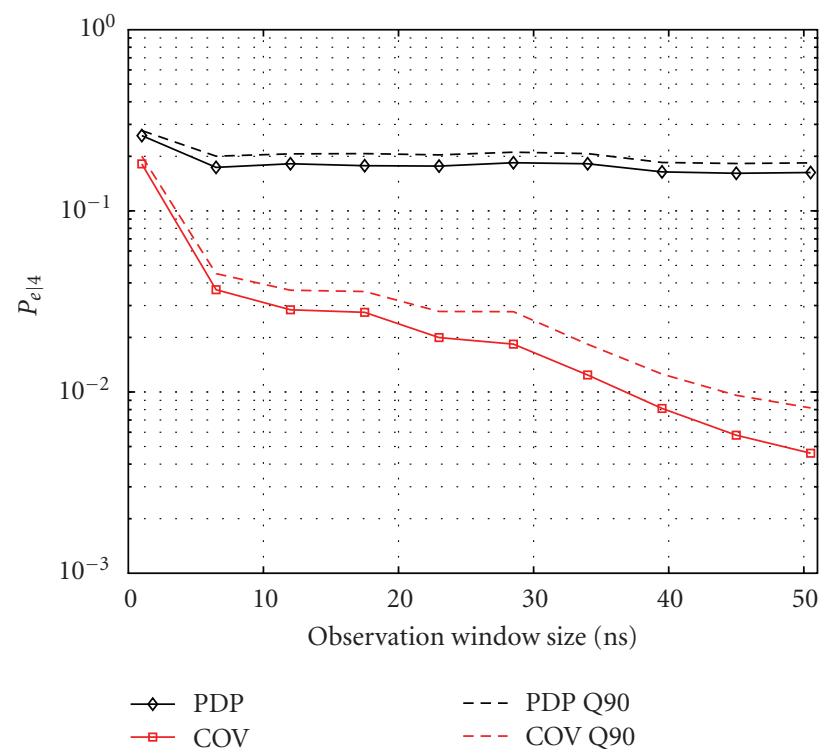

(a)

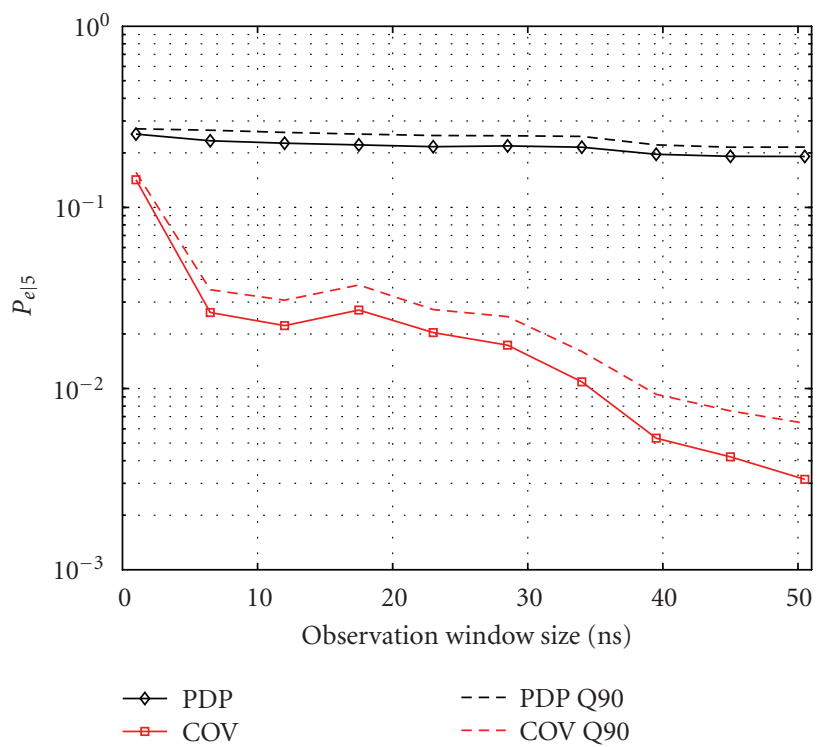

(b)

FiguRE 10: Region pair $(4,5)$ with measured CIRs at SNR $=25 \mathrm{~dB}$ for increasing observation window.

introduced. A theoretical framework consisting of probability models and maximum likelihood algorithms with different performances and complexities has been developed. Based on this framework, an analytic study of the error probabilities of the binary hypothesis-testing problem has been performed. The feasibility and fundamental performance dependencies on design space parameters of UWB geo-regioning have been evaluated by applying the derived algorithms to measured CIRs.

The presented performance results reveal the superiority of the algorithms assuming correlated CIR taps over those assuming independent taps. This implies that, for UWB georegioning, the reflectors and scatterers are correlated, and the channel tap correlations are region dependent. The drawback of the correlation is the increased number of model parameters, which must be estimated from a priori data. However, this number can be significantly reduced by adjusting system parameters like bandwidth and observation window. For example, UWB geo-regioning can achieve probabilities of misclassification of less than $10^{-2}$ for a CIR length of 20 taps observing $10 \mathrm{~ns}$ at a bandwidth of $2 \mathrm{GHz}$, provided that the SNR is around $25 \mathrm{~dB}$ and not two neighboring regions are 


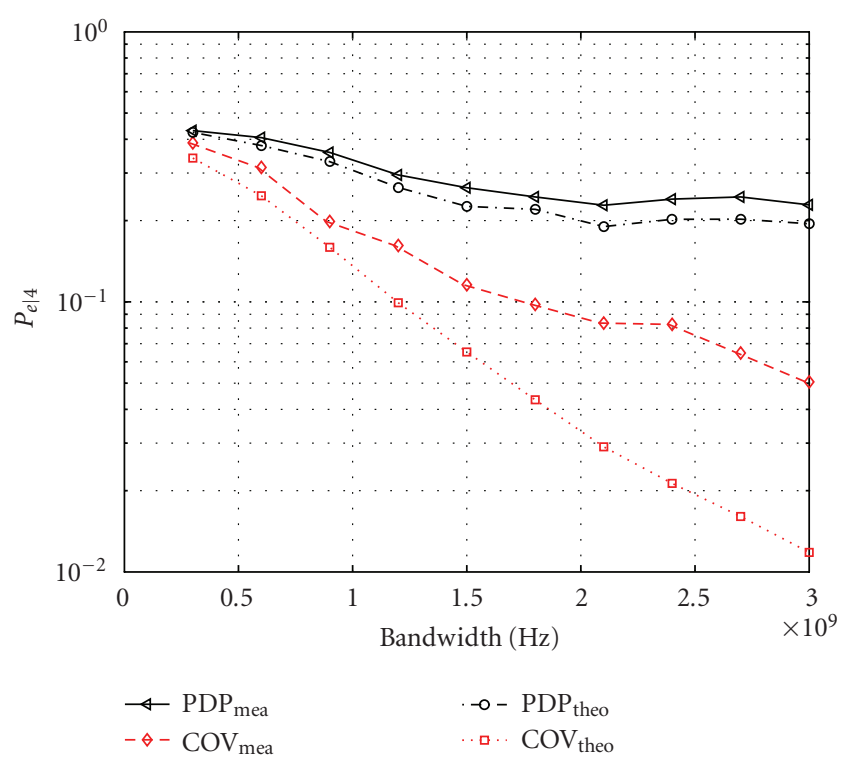

(a)

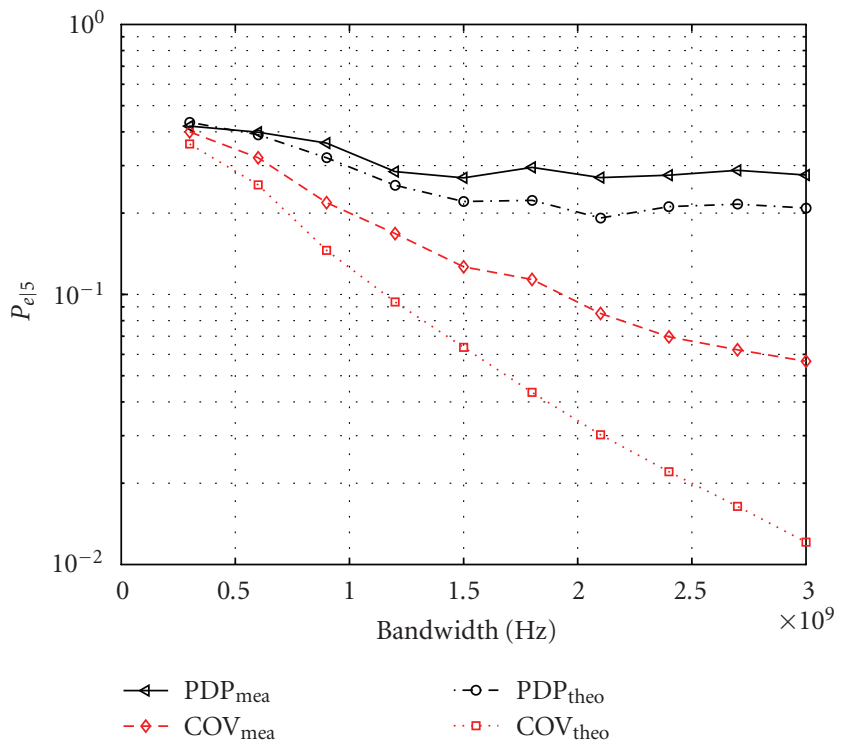

(b)

Figure 11: Region pair $(4,5)$ at $\mathrm{SNR}=25 \mathrm{~dB}$ for increasing bandwidth.

considered. In this case, around 40 a priori CIRs are required to achieve reliable parameter estimates. For the classification (normal receiver operation), only the 20 tap CIRs from different UWB transmitters must be estimated, which can be done with reasonable receiver complexity. Moreover, these classified CIRs can be used to increase the accuracy of the parameter estimates depending on a soft indicator, such as the likelihood value.

Future work will address the ad-hoc problem of generating clusters and regions out of a set of CIRs, where bootstrap methods are promising candidates. Another interesting aspect is the exploitation of different CIRs as natural spreading sequences given by the physical propagation environment and transmitter and receiver locations. Here, it would be most beneficial to have a distance measure for instantaneous CIRs. This idea in conjunction with UWB georegioning could help to relax the multiaccess problem in dense wireless networks.

\section{ACKNOWLEDGMENT}

The work presented in this paper was partially supported by the National Competence Center in Research on Mobile Information and Communication Systems (NCCR-MICS), a center supported by the Swiss National Science Foundation under Grant no. 5005-67322.

\section{REFERENCES}

[1] S. Gezici, Z. Tian, G. B. Giannakis, et al., "Localization via ultra-wideband radios: a look at positioning aspects of future sensor networks," IEEE Signal Processing Magazine, vol. 22, no. 4 , pp. $70-84,2005$.
[2] J.-Y. Lee and R. A. Scholtz, "Ranging in a dense multipath environment using an UWB radio link," IEEE Journal on Selected Areas in Communications, vol. 20, no. 9, pp. 1677-1683, 2002.

[3] B. Denis, J. Keignart, and N. Daniele, "Impact of NLOS propagation upon ranging precision in UWB systems," in Proceedings of IEEE Conference on Ultra Wideband Systems and Technologies (UWBST'03), pp. 379-383, Reston, Va, USA, November 2003.

[4] Y. Qi, H. Suda, and H. Kobayashi, "On time-of-arrival positioning in a multipath environment," in Proceedings of the 60th IEEE Vehicular Technology Conference (VTC'04), vol. 60, no. 5, pp. 3540-3544, Los Angeles, Calif, USA, September 2004.

[5] P. Bahl and V. N. Padmanabhan, "RADAR: an in-building RFbased user location and tracking system," in Proceedings of 19th Annual Joint Conference of the IEEE Computer and Communications Societies (INFOCOM '00), vol. 2, pp. 775-784, Tel Aviv, Israel, March 2000.

[6] C. Nerguizian, C. Despins, and S. Affès, "Geolocation in mines with an impulse response fingerprinting technique and neural networks," IEEE Transactions on Wireless Communications, vol. 5, no. 2, pp. 603-611, 2006.

[7] R. Vaughan and J. B. Andersen, Channels, Propagation and Antennas for Mobile Communications, The Institiution of Electrical Engineers, London, UK, 2003.

[8] D. Cassioli, M. Z. Win, and A. F. Molisch, "The ultra-wide bandwidth indoor channel: from statistical model to simulations," IEEE Journal on Selected Areas in Communications, vol. 20, no. 6, pp. 1247-1257, 2002.

[9] J. Keignart and N. Daniele, "Channel sounding and modelling for indoor UWB communications," in Proceedings of International Workshop on Ultra Wideband Systems(IWUWBS '03), Oulu, Finland, June 2003.

[10] P. Pagani, P. Pajusco, and S. Voinot, "A study of the ultrawide band indoor channel: propagation experiment and measurement results," in Proceedings of International Workshop on Ultra Wideband Systems(IWUWBS '03), Oulu, Finland, June 2003. 
[11] U. G. Schuster and H. Bölcskei, "Ultrawideband channel modeling on the basis of information-theoretic criteria," IEEE Transactions on Wireless Communications, vol. 6, no. 7, pp. 2464-2474, 2007.

[12] T. Anderson, An Introduction to Multivariate Statistical Analysis, John Wiley \& Sons, New York, NY, USA, 3rd edition, 2003.

[13] M. Schwartz, Communication Systems and Techniques, McGraw-Hill, New York, NY, USA, 1st edition, 1966.

[14] J. G. Proakis, Digital Communications, McGraw-Hill, New York, NY, USA, 4th edition, 2001.

[15] F. Althaus, F. Troesch, T. Zasowski, and A. Wittneben, "STS measurements and characterization," PULSERS Deliverable D3b6a, vol. IST-2001-32710 PULSERS, 2005.

[16] C. Steiner and A. Wittneben, "UWB geo-regioning using multivariate channel statistics," in Proceedings of IEEE International Conference on Communications(ICC '07), pp. 57635768, Glasgow, Scottland, June 2007.

[17] C. M. Bishop, Neural Networks for Pattern Recognition, Clarendon Press, Oxford, UK, 1st edition, 1995.

[18] J. Kunisch and J. Pamp, "Measurement results and modeling aspects for the UWB radio channel," in Proceedings of IEEE Conference on Ultra Wideband Systems and Technologies (UWBST'02), pp. 19-23, Baltimore, Md, USA, May 2002.

[19] J. Karedal, S. Wyne, P. Almers, F. Tufvesson, and A. Molisch, "UWB channel measurements in an industrial environment," in Proceedings of IEEE Global Telecommunications Conference (GLOBECOM '04), vol. 6, pp. 3511-3516, Dallas, Tex, USA, November 2004. 